\title{
Multifractal analysis of inclined oil-water countercurrent flow
}

\author{
Zhu Lei, Jin Ningde*, Gao Zhongke and Zong Yanbo \\ School of Electrical Engineering and Automation, Tianjin University, Tianjin 300072, China \\ (C) China University of Petroleum (Beijing) and Springer-Verlag Berlin Heidelberg 2014
}

\begin{abstract}
Characterizing countercurrent flow structures in an inclined oil-water two-phase flow from one-dimensional measurement is of great importance for model building and sensor design. Firstly, we conducted oil-water two-phase flow experiments in an inclined pipe to measure the conductance signals of three typical water-dominated oil-water flow patterns in inclined flow, i.e., dispersion oil-in-water pseudo-slug flow (PS), dispersion oil-in-water countercurrent flow (CT), and transitional flow (TF). In pseudo-slug flow, countercurrent flow and transitional flow, oil is completely dispersed in water. Then we used magnitude and sign decomposition analysis and multifractal analysis to reveal levels of complexity in different flow patterns. We found that the PS and CT flow patterns both exhibited high complexity and obvious multifractal dynamic behavior, but the magnitude scaling exponent and singularity of the CT flow pattern were less than those of the PS flow pattern; and the TF flow pattern exhibited low complexity and almost monofractal behavior, and its magnitude scaling was close to random behavior. Meanwhile, at short time scales, all sign series of two-phase flow patterns exhibited very similar strong positive correlation; at high time scales, the scaling analysis of sign series showed different anti-correlated behavior. Furthermore, with an increase in oil flow rate, the flow structure became regular, which could be reflected by the decrease in the width of spectrum and the difference in dimensions. The results suggested that different oil-water flow patterns exhibited different nonlinear features, and the varying levels of complexity could well characterize the fluid dynamics underlying different oil-water flow patterns.
\end{abstract}

Key words: Inclined oil-water two-phase flow, magnitude correlation, multifractal spectrum, nonlinear dynamics

\section{Introduction}

In the petroleum industry, oil is often produced and transported together with water, and the complex interfaces of two fluids can generate different flow patterns with various characteristic distributions. Important applications in the petroleum industry such as oil production engineering and predictions of liquid holdup and pressure drop strongly depend on the flow patterns. Especially in an inclined oilwater two-phase flow, countercurrent flow occurs widely. Due to the existence of complex flow structures and the local velocity distribution, computational fluid dynamics approaches are usually unable to describe inclined oilwater countercurrent flow patterns. Therefore, the dynamic characterization of inclined oil-water two-phase flow is an important problem of significant challenge.

Earlier investigations into inclined oil-water two-phase flow were mainly focused on semi-empirical and semitheoretical methods. For example, Hill and Oolman (1982) pointed out, in 152-mm ID pipes, the most troublesome effect

*Corresponding author. email: ndjin@tju.edu.cn

Received April 12, 2013 of well deviation on production logging tool responses was the non-uniform phase distribution across the pipe. They observed a type of segregated flow pattern where the water phase occupied most of the pipe and a steady reverse flow of water occurred along the bottom of the pipe; however, a small change in the deviation angle can cause a large change in the velocity profile distribution. Flores et al (1999) conducted a comprehensive experimental study of inclined oil-water flow with 50.8-mm ID pipes, and summarized seven flow patterns with four water-dominated flow patterns, two oil-dominated flow patterns and a transitional flow pattern. Gong et al (2007) discussed the factors of critical conditions for phase inversion of heavy oil in water two-phase flow, and especially studied the effect of mixture velocity on the water fraction for phase inversion. Yang et al (2008) obtained flow pattern maps with invading logging tools for inclined oil-water two-phase flow, and analyzed the effects of inclination and production logging. Kumara et al (2010) investigated the oil-water flow structure in slightly inclined pipes with a inclination from $5^{\circ}$ upward to $5^{\circ}$ downward by particle image velocimetry (PIV), and the results showed the velocity and turbulence profiles were strongly affected by the pipe inclination. Strazza et al (2011) focused on the pressure drops and oil hold-up 
measurements in horizontal and slightly inclined pipes, and they found that the classical models agreed well with the experimental data. Wang et al (2012) proposed an algorithm to solve the difficulty in computation of transient pressure distribution of slanted wells with any inclination angle. Rodriguez and Baldani (2012) presented pressure gradient and hold up data for horizontal and inclined oil-water wavy stratified flow, and the results of phenomenological models and predictions were compared with experimental data.

Recently, nonlinear time series analysis has made great progress. The detrended fluctuation analysis (DFA) method proposed by Peng et al (1995) has been widely used to detect long-range correlation and power-law properties in nonstationary time series. Ashkenazy et al (2001) proposed a method based on magnitude and sign decomposition to analyze heartbeat signals with long-range correlation and found that a time series with identical correlation properties may have completely different time ordering, which can be characterized by different scaling exponents for the magnitude and sign series. Moreover, the long-range correlations of magnitude series indicated that the nonlinear behavior and the sign time series mainly related to linear properties of the original series. Kantelhard et al (2002) extended the DFA method to propose multifractal detrended fluctuation analysis (MF-DFA). The MF-DFA can be used not only to explore the long-range correlation and scaling invariance, but also to compute the roughness exponent and identify the multifractal property in non-stationary time series.

Although some progress has been made in applying nonlinear analysis methods to study inclined oil-water twophase flow (Daw et al, 1995; Gao et al, 2010; Zhu et al, 2011), there still exist limitations as how to characterize the dynamic characteristics of countercurrent flow from experimental signals. Inclined oil-water two-phase flow contains abundant nonlinear dynamic characteristics, and usually has complex fluid structures. Especially, countercurrent flow structures exist due to the effect of gravity and methods such as numerical modeling have limitations for uncovering the dynamic characteristics of countercurrent flow. The measured signals usually contain a large number of linear and nonlinear components, so it is hard to characterize dynamic behavior of inclined oil-water two-phase flow by only one scaling. Note that, the previous studies mainly focused on flow pattern identification and did not pay much attention to dynamic characteristic discrepancies for the same kind of flow pattern.

Based on the above mentioned, we conducted inclined oil-water two-phase flow experiments to measure the conductance signals of three typical water-dominated flow patterns, i.e., pseudo-slug (PS), countercurrent (CT), and transitional flow (TF). We first decompose nonlinear and linear components from the original time series by magnitude and sign decomposition analysis. Then we extract scaling exponents of singular spectra and their change under different flow conditions. Our long-range correlation and multifractal analysis provide important information for understanding the nonlinear dynamic mechanisms underlying the transitions of different flow patterns.

\section{Methods}

\subsection{Magnitude and sign decomposition method}

The magnitude and sign of the original signals measured from physical processes contain lots of important information, which can characterize the dynamic fluctuation and reflect the underlying behavior in a given system. So we introduce the magnitude and sign decomposition method to explore fluid characteristics from the time series. The magnitude and sign decomposition analysis consists of the following steps:

Firstly, the increment series is generated by $\Delta s(i)=s(i+1)-s(i)$, where $s(i)$ is the original signal. $\Delta s(i)$ can be decomposed into a magnitude series $|\Delta s(i)|$ and a sign series $\operatorname{sgn} \Delta s(i)$. A positive increment is represented by sign $(+1)$, while a negative increment is represented by sign $(-1)$. We choose sign (0) to represent a special case of $\Delta s(i)=0$. To avoid the limitation in the accuracy of anti-correlation, we subtract from the magnitude and sign series their average and then integrate both series.

Secondly, the DFA-2 analysis should be used on both magnitude and sign series, and we can obtain corresponding scaling exponents for magnitude and sign series by calculating the slope of $F(n) / n$ in double logarithmic coordinates.

For the original signal, the long-term nonlinear properties relate to the magnitude series with power-law correlation, and the linear features are indicated by the sign series (Ashkenazy et al, 2001). Otherwise, the width of the multifractal spectrum relates to the value of the magnitude scaling exponent. So this method can be used to obtain nonlinear characteristics in short non-stationary signals.

\subsection{Multifractal detrended fluctuation analysis}

Multifractal features probably exist in inclined oil-water two-phase countercurrent flow. To probe the multifractal characteristics of measured signals, we apply MF-DFA. The method is not only frequently used to detect long-range correlation and scaling invariance, but also used to identify multifractal properties in non-stationary time series. The MFDFA algorithm can be described as follows (Kantelhard et al, 2002):

Firstly, given an original signal $\left\{x_{k}\right\}$, where $k=1,2, \cdots$, $N$, and $N$ is the length of the series, we generate a new time series:

$$
Y(i)=\sum_{k=1}^{i}\left[x_{k}-\bar{x}\right], \quad i=1,2, \cdots, N
$$

where $\bar{x}$ is the mean of $\left\{x_{k}\right\}$.

Then, the new signal $Y(i)$ is partitioned into $N_{r}$ disjoint segments of the same size $r$, where $N_{r}=\operatorname{int}(N / r)$. Since $N$ is often not multiple of the segment size $r$, some data at the end of the time series may remain and will be ignored by this way. In order to take these ending parts of the signal into consideration, the same partitioning procedure can be repeated starting from the ending. Then there will be $2 N_{r}$ segments and calculating the average over them can eliminate the boundary influence. 
For each segment the trend of data can be determined by a bivariate polynomial function $y_{y}(i)$. Then, the detrended fluctuation function $F(v, r)$ can be calculated by:

$$
\begin{gathered}
F^{2}(v, r)=\frac{1}{r} \sum_{i=1}^{r}\left\{Y[(v-1) r+i]-y_{v}(i)\right\}^{2} \quad i=1,2, \cdots, N_{r} \\
F^{2}(v, r)=\frac{1}{r} \sum_{i=1}^{r}\left\{Y\left[N-\left(v-N_{r}\right) r+i\right]-y_{v}(i)\right\}^{2} \\
i=N_{r}, \cdots, 2 N_{r}
\end{gathered}
$$

and we use a polynomial fit of order 2 in this paper.

The $F_{q}(r)$ is calculated by averaging over all the segments:

$$
F_{q}(r)=\frac{1}{2 N_{r}}\left\{\sum_{v=1}^{2 N_{r}}\left[F^{2}(v, r)\right]^{\frac{q}{2}}\right\}^{\frac{1}{q}}
$$

where $q$ is the value of order, which can take any real value except zero. When $q=0$, it should be defined as:

$$
F_{0}(r)=\exp \left\{\frac{1}{4 N_{r}} \sum_{v=1}^{2 N_{r}} \ln \left[F^{2}(v, r)\right]^{2}\right\}
$$

Varying the value of $q$, we can obtain the scaling relation between the fluctuation function $F_{q}(r)$ and the size scale $r$, as a power-law:

$$
F_{q}(r) \sim r^{h(q)}
$$

where the exponent $h(q)$ is called the generalized Hurst exponent. For a given system, the monofractal feature relates to a constant scaling exponent $h(q)$, while the multifractal feature relates to a nonlinear function of $h(q)$.

The multifractal property can be characterized by the mass exponents $\tau(q)$, which also is a nonlinear function. The $\tau(q)$ can be calculated through:

$$
\tau(q)=q h(q)-D_{f}
$$

where $D_{f}$ is the geometric support dimension.

Then, we can obtain the singularity strength function $\alpha(q)$ and the singularity spectrum $f(\alpha)$ by Legendre transform as follows:

$$
\left\{\begin{array}{l}
\alpha(q)=\tau^{\prime}(q) \\
f(\alpha)=q \alpha(q)-\tau(q)
\end{array}\right.
$$

\section{Experimental facility and data acquisition}

The inclined oil-water two-phase flow experiments were carried out in the Multiphase Flow Laboratory of Tianjin University. Details about the flow facility are described in our paper (Jin et al, 2008). The experimental media were tap water and No.15 industry white oil with a surface tension of $0.035 \mathrm{~N} / \mathrm{m}$ and a viscosity of $12.0 \mathrm{mPa} \cdot \mathrm{s}\left(40{ }^{\circ} \mathrm{C}\right)$. In the inclined upward $125-\mathrm{mm}$ ID pipes at inclination $45^{\circ}$, the water flow rates were $0.21,0.42,0.83$ and $1.64 \mathrm{~m}^{3} / \mathrm{h}$ and the oil flow rates ranged from 0.53 to $8.3 \mathrm{~m}^{3} / \mathrm{h}$. Based on flow pattern definition proposed by Flores et al (1999), we had observed three typical different water-dominated flow patterns in the experiment including PS, CT and TF, whose schematic diagrams are shown in Fig. 1. The conductance signals were measured with a vertical multi-electrode array (VMEA) conductance sensor (Jin et al, 2008) with a sampling frequency of $400 \mathrm{~Hz}$, and typical signals are shown in Fig. 2. The signals were recorded by National Instrument Corporation's data acquisition cards PXI 4472 and PXI 6115 operated by LabVIEW software.

Under different flow rates and oil volume fractions, the different flow structures with different conductance paths and liquid equivalent conductivity will respectively cause voltage fluctuation on the measuring electrode, due to significant differences in electrical properties between oil and water phases. Based on the measured signals, we investigated the long-range correlation and levels of complexity of different inclined oil-water flow patterns by using the magnitude and sign decomposition analysis and multifractal analysis.

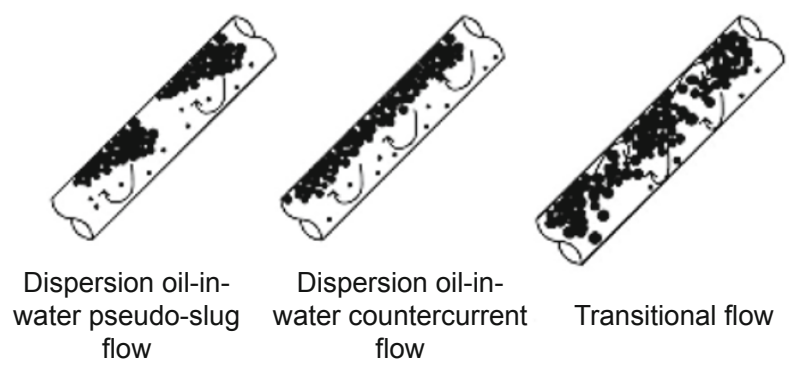

Fig. 1 Schematic diagrams of three typical inclined oil-water two-phase flow patterns

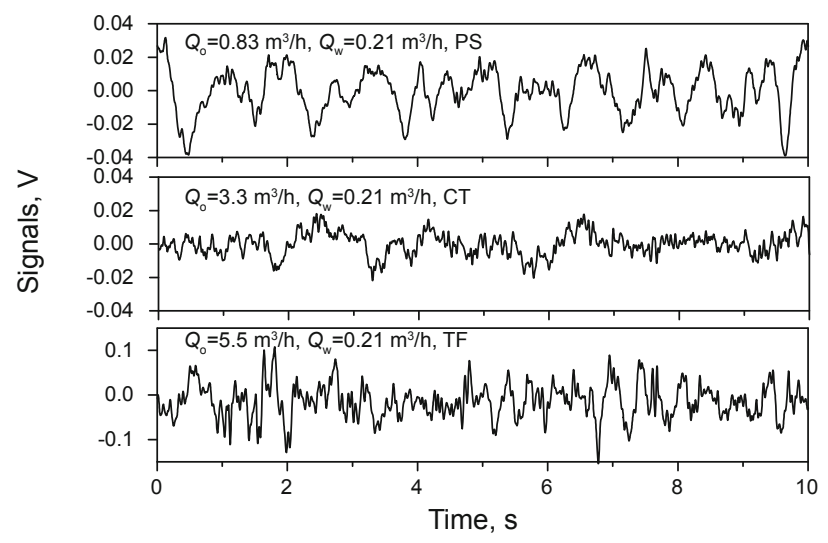

Fig. 2 Fluctuating conductance signals of three typical inclined oil-water two-phase flow patterns

\section{Magnitude and sign scaling in inclined oil- water two-phase countercurrent flow signals}

Ashkenazy et al (2001) demonstrated the effectiveness of the magnitude and sign series in the analysis of long-range correlations. In fact, the long-range correlations of magnitude series can indicate the nonlinear behavior of the original series and the sign time series mainly reflect their linear 
properties.

Our results show that the magnitude series of different flow patterns all exhibit strong positive correlations which can be characterized by $\alpha_{\text {mag }}>0.5$, as shown in Fig. 3(a), indicating nonlinear features in flow mechanisms. For the same flow pattern with different flow conditions, the variation tendency of the magnitude series is basically similar and the corresponding scaling exponent is similar as well; while the magnitude scaling exponents of different flow patterns are distinctly different, this indicates the nonlinear dynamics characteristics for PS, CT and TF flow patterns.

Correspondingly as shown in Fig. 3(b), at short time scales, all sign series of inclined oil-water flow patterns show very strong positive correlation, and the exponents for different flow patterns are very similar; at long time scales, the DFA scaling analysis of the sign series shows different anti-correlated behavior $(\alpha<0.5)$ for different flow patterns. For all water-dominated flow patterns, the variation tendencies of the sign series are similar; while the sign scaling exponents of different flow patterns are different at long time scales, which indicates the linear dynamic characteristics of PS, CT and TF flow patterns.
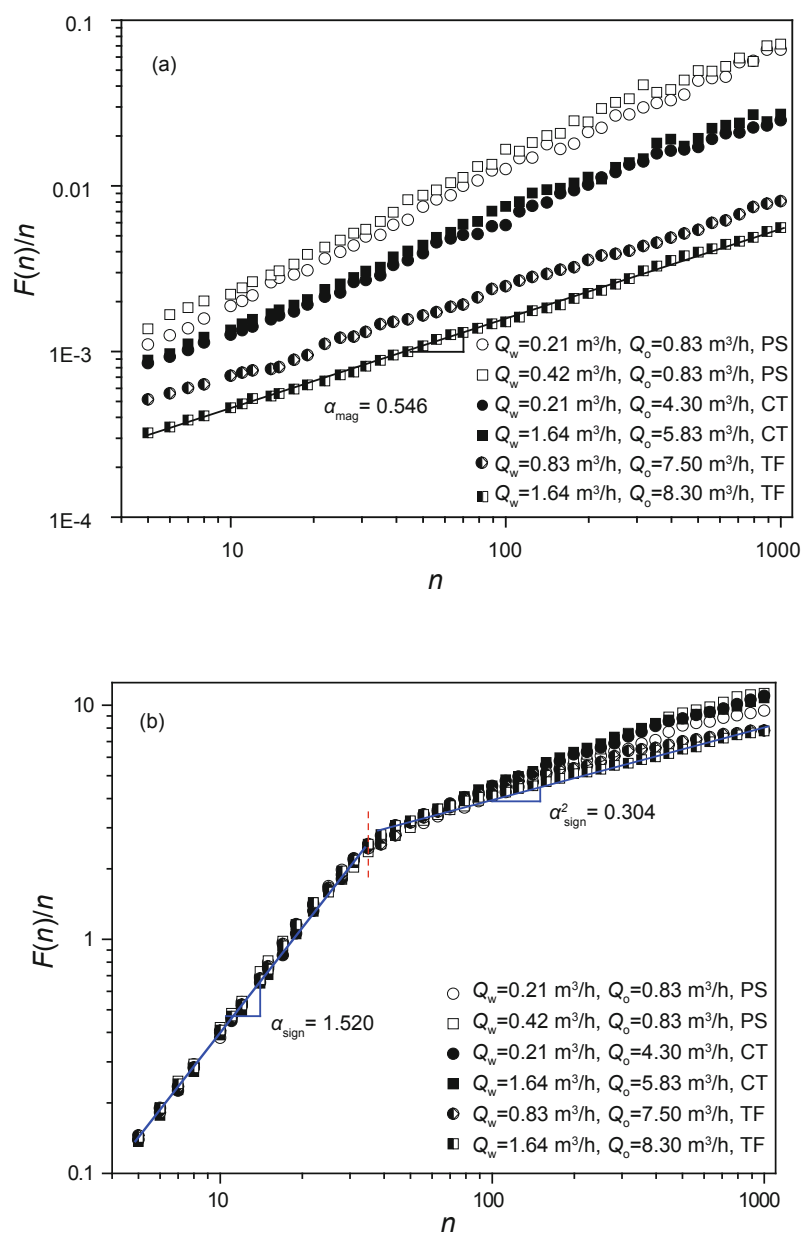

Fig. 3 Magnitude and sign scaling properties of water-dominated flow patterns (a) DFA analysis of magnitude series and (b) sign series
To further explore the correlation of flow patterns for different time scales, we calculate the magnitude scaling exponents $\alpha_{\text {mag }}$ over a broad range of time scales $5<n<1000$; for sign scaling, we calculate the short-range regime for time scale $5<n<34$ with scaling exponent $\alpha_{\text {sign }}^{1}$, and the long-range regime for time scale $34<n<1000$ with scaling exponent $\alpha_{\text {sign }}^{2}$. For each measure scaling, the group average \pm 1 standard deviation is presented.

Table 1 Results of magnitude and sign DFA analysis of inclined oil-water two-phase flow

\begin{tabular}{cccc}
\hline Flow pattern & $\alpha_{\text {mag }}$ & $\alpha_{\text {sign }}^{1}$ & $\alpha_{\text {sign }}^{2}$ \\
\hline PS & $0.74 \pm 0.03$ & $1.52 \pm 0.03$ & $0.45 \pm 0.05$ \\
CT & $0.67 \pm 0.01$ & $1.52 \pm 0.02$ & $0.43 \pm 0.01$ \\
TF & $0.53 \pm 0.01$ & $1.52 \pm 0.01$ & $0.32 \pm 0.02$ \\
\hline
\end{tabular}

As we can see in Table 1, the range of $\alpha_{\text {mag }}$ is different for all flow patterns. Previous studies have demonstrated that information about nonlinear properties of flow dynamics can be quantified by long-range power-law correlation in magnitude of the increments in fluctuation signals, so the different magnitude scaling exponents exhibit the different nonlinear dynamics of flow patterns.

The mean value of magnitude scaling exponents of PS is $\alpha_{\text {mag }}=0.74 \pm 0.03$, which is larger than other flow patterns, indicating that its long-range correlation is the strongest among three types of water-dominated flow patterns. For the PS flow pattern, the oil phase move fast in the upward direction as the intermittent oil swarms structure, while countercurrent water flow exists at the bottom of the pipe due to the effects of pressure, viscosity and gravity components in the opposite direction of the main flow. The intermittent quasi-periodic characteristics in the flow structure of PS flow determine the strongest long-range correlation.

For the CT flow pattern, the mean value of magnitude scaling exponents is $\alpha_{\mathrm{mag}}=0.67 \pm 0.01$, which is larger than the TF pattern but smaller than the PS flow pattern. The intervals between oil swarms become shorter, and then disappear with an increase in oil flow rate, and the CT flow pattern occurs. On the other hand, counter flow of some oil droplets will appear near the interface between the oil and water phases, which is caused by countercurrent water flow in the bottom of the pipe. So the positive correlation of the CT flow pattern is weaker than the PS flow pattern.

The mean value of magnitude scaling exponents of TF is $\alpha_{\operatorname{mag}}=0.53 \pm 0.01$, indicating its behavior close to random. For the TF flow pattern, the flow structure contains three parts: the thin oil film at the top; local water-countercurrent flow at the bottom; and alternation of oil-dominated and waterdominated flow structures in the middle of the pipe. It should be pointed that, as an unsteady transitional flow pattern, the most significant features of TF are the alternations of oildominated and water-dominated flow structure, which is consistent with our experiments. Such flow structure indicates 
random flow behavior resulting in all magnitude scaling exponents close to 0.5 . The corresponding result for its property will be discussed in the next section. Otherwise, the latter analysis would show the magnitude scaling exponents relate to the width of the multifractal spectrum of the original time series. In this regard, the width of the multifractal spectrum increases with the increase in the magnitude scaling exponent between 0.5 and $1\left(0.5<\alpha_{\text {mag }}<1\right)$, but not in a monotonous form (Ashkenazy et al, 2001)).

The results show that the time series composed of the sign of the increments in the original signal contain information about the underlying dynamics, which is necessarily complementary to the original and the magnitude series. In the long-range region, the sign scaling behavior of PS, CT and TF flow patterns are different, reflecting that the intrinsic dynamics of the three flow patterns are different (Peng et al, 1995). The sign scaling exponent of the PS flow pattern varies in a larger range $\left(\alpha_{\text {sign }}^{2}=0.45 \pm 0.05\right)$ because this flow pattern occurs across a wide region from low to moderate oil and water superficial velocities. With an increase in the oil flow rate, the interval between oil swarms will become shorter and shorter, so part of the sign scaling exponents of the PS flow pattern cover those of the CT flow pattern $\left(\alpha_{\text {sign }}^{2}=0.43 \pm 0.01\right)$.
The sign scaling property of the TF flow pattern shows stronger anti-correlation $\left(\alpha_{\text {sign }}^{2}=0.32 \pm 0.02\right)$, indicating the oscillatory characteristics of the fluctuating signals for this flow pattern.

\section{Multifractal properties in inclined oil- water two-phase flow signals}

\subsection{Multifractal spectrum of inclined oil-water two- phase flow}

The fractal dimension $f(\alpha)$, which is the function of singularity strength $\alpha$, can be defined as the multifractal spectrum or singularity spectrum. So, $\alpha$ and $f(\alpha)$ are two most important characteristics used to characterize the multifractal. We have obtained the values of $\alpha(q)$ and $f(\alpha)$ through the Legendre transform from $h(q)$ and $\tau(q)$ for all the experimental flow conditions. Kantelhardt et al (2002) proposed the multifractal analysis method and took the monofractal series, binomial multifractal series and random cascade model as examples to demonstrate that this method can represent the complexity of different dynamic systems. Figs. 4-7 illustrate the distributions of $f(\alpha)$ with respect to $\alpha$ for the PS, CT and TF flow patterns with different $Q_{\mathrm{o}}$ when
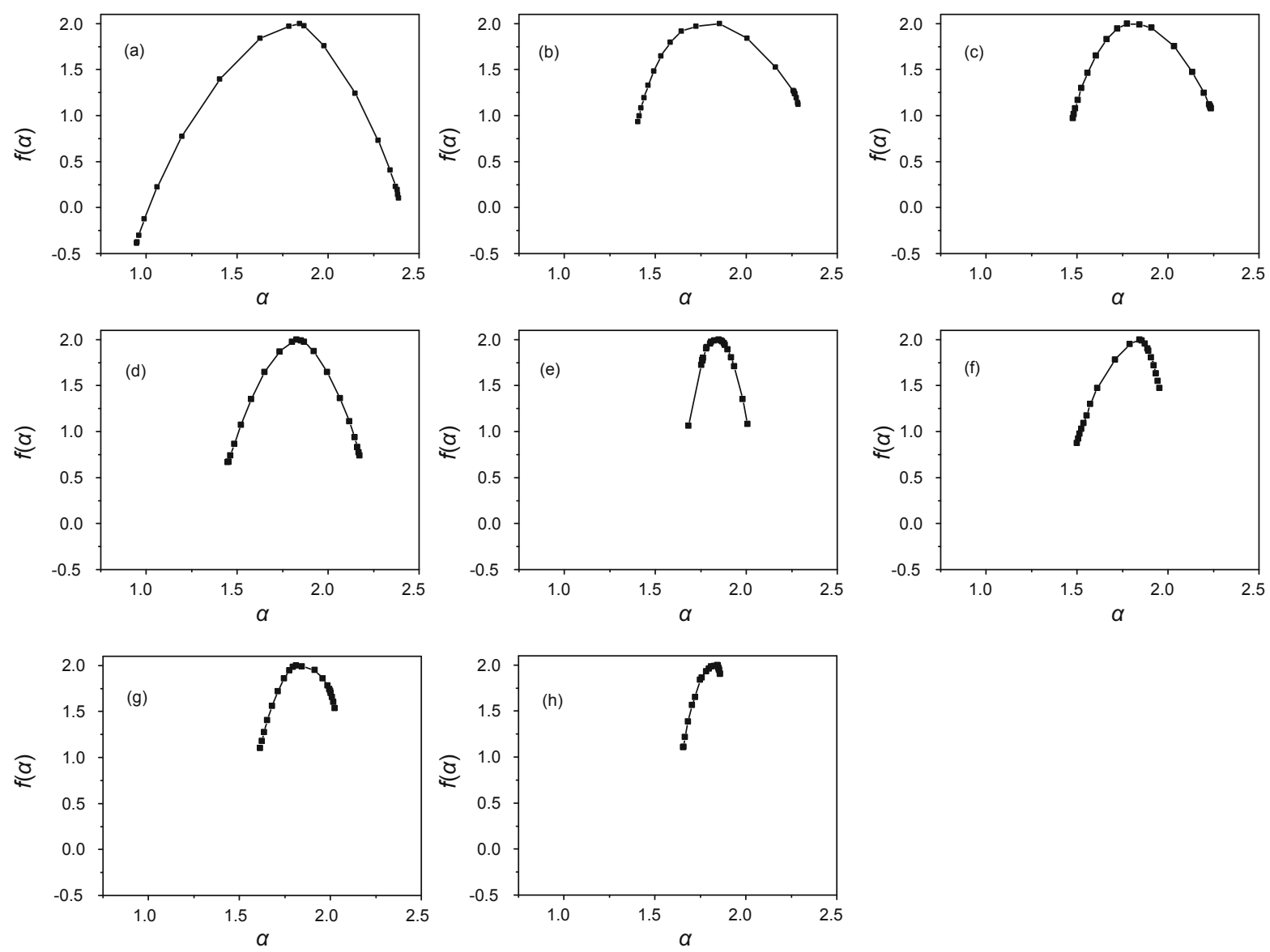

Fig. 4 Multifractal spectra versus $Q_{\mathrm{o}}$ at inclination $45^{\circ}$ and $Q_{\mathrm{w}}=0.21 \mathrm{~m}^{3} / \mathrm{h}$

(a) $Q_{\mathrm{o}}=0.53 \mathrm{~m}^{3} / \mathrm{h}, \mathrm{PS}$; (b) $Q_{\mathrm{o}}=0.83 \mathrm{~m}^{3} / \mathrm{h}, \mathrm{PS}$; (c) $Q_{\mathrm{o}}=1.25 \mathrm{~m}^{3} / \mathrm{h}, \mathrm{PS}$; (d) $Q_{\mathrm{o}}=1.62 \mathrm{~m}^{3} / \mathrm{h}, \mathrm{PS}$;

(e) $Q_{\mathrm{o}}=2.50 \mathrm{~m}^{3} / \mathrm{h}$, PS; (f) $Q_{\mathrm{o}}=3.30 \mathrm{~m}^{3} / \mathrm{h}, \mathrm{CT}$; (g) $Q_{\mathrm{o}}=4.30 \mathrm{~m}^{3} / \mathrm{h}, \mathrm{CT}$; (h) $Q_{\mathrm{o}}=5.50 \mathrm{~m}^{3} / \mathrm{h}$, TF 

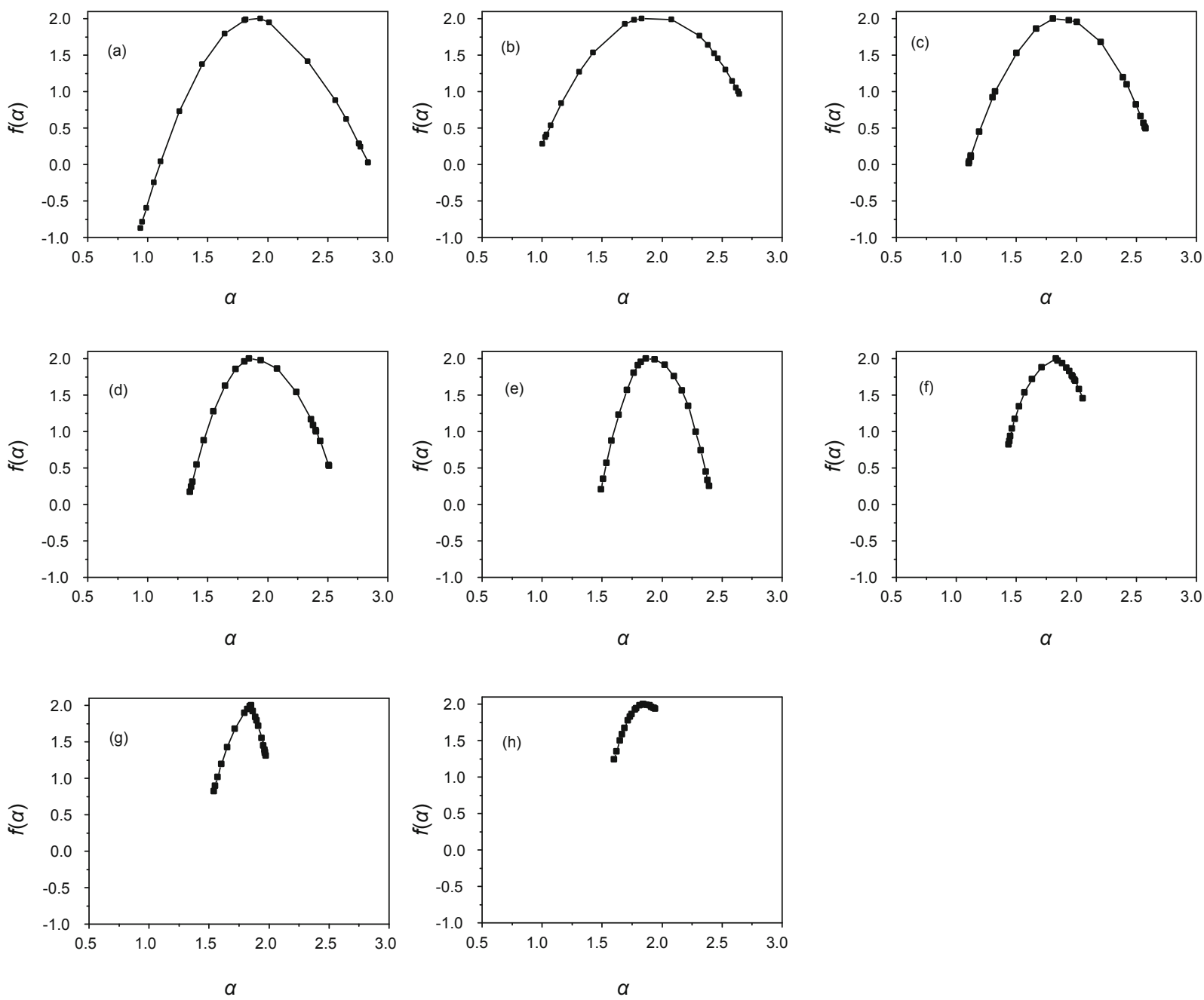

Fig. 5 Multifractal spectra versus $Q_{\mathrm{o}}$ at inclination $45^{\circ}$ and $Q_{\mathrm{w}}=0.42 \mathrm{~m}^{3} / \mathrm{h}$

(a) $Q_{\mathrm{o}}=0.42 \mathrm{~m}^{3} / \mathrm{h}, \mathrm{PS}$; (b) $Q_{\mathrm{o}}=0.83 \mathrm{~m}^{3} / \mathrm{h}, \mathrm{PS}$; (c) $Q_{\mathrm{o}}=1.67 \mathrm{~m}^{3} / \mathrm{h}, \mathrm{PS}$; (d) $Q_{\mathrm{o}}=2.50 \mathrm{~m}^{3} / \mathrm{h}, \mathrm{PS}$;

(e) $Q_{\mathrm{o}}=3.30 \mathrm{~m}^{3} / \mathrm{h}$, PS; (f) $Q_{\mathrm{o}}=4.17 \mathrm{~m}^{3} / \mathrm{h}, \mathrm{CT}$; (g) $Q_{\mathrm{o}}=5.80 \mathrm{~m}^{3} / \mathrm{h}, \mathrm{CT}$; (h) $Q_{\mathrm{o}}=7.50 \mathrm{~m}^{3} / \mathrm{h}, \mathrm{TF}$

$Q_{\text {w }}$ is fixed at $0.21,0.42,0.83$ and $1.64 \mathrm{~m}^{3} / \mathrm{h}$, respectively.

As can be seen, the multifractal spectra of different flow patterns are smooth curves with a peak value. The maximum of $f(\alpha)$ is 2 , which is equal to the geometric support of the fractal measure for the research object, and the peak appears near $\alpha=1.85$. An intriguing feature in Figs. 4(a)-(7a) is that the $f(\alpha)$ function for the PS flow pattern becomes negative when $\alpha$ is smaller than about 1.0. The negative dimension $(f(\alpha)<0)$ was investigated in several experiments such as the diffusionlimited aggregation (Amitrano et al, 1986) and the energy dissipation field of turbulent flows (Chhabra and Sreenivasan, 1991). The negative dimension describes rarely occurring events (Mandelbrot, 1990) and one needs an exponentially increasing number of samples to observe the subsets with the same a value (Chhabra and Sreenivasan, 1991).

The multifractal spectra for different flow patterns can directly exhibit different shapes, among which PS flow exhibits nearly symmetric spectrum shape and CT flow exhibits a left-hooked shape. The range of multifractal spectra for TF is very narrow, which to some extent means that this flow pattern is close to monofractal, which is consistent to the magnitude scaling exponent in the former chapter. According to Figs. 4-7, the multifractal spectrum shapes of different flow conditions even for the same flow pattern are not completely the same. In addition, the multifractal spectra for different flow conditions are very sensitive to the change of flow rate, which means the multifractal spectrum could be a potentially useful tool for analyzing nonlinear mechanisms underlying the transitions of different flow patterns.

The minimum singularity $\alpha_{\min }$ and the maximum singularity $\alpha_{\max }$, which respectively indicate the least and most singular, can be calculated:

$$
\left\{\begin{array}{l}
\alpha_{\min }=\lim _{q \rightarrow+\infty} \frac{\mathrm{d} \tau(q)}{\mathrm{d} q} \\
\alpha_{\max }=\lim _{q \rightarrow-\infty} \frac{\mathrm{d} \tau(q)}{\mathrm{d} q}
\end{array} \quad q \rightarrow \pm \infty\right.
$$

The corresponding parameters $f\left(\alpha_{\min }\right)$ and $f\left(\alpha_{\max }\right)$ reflect the fractal dimensions characterized by $\alpha=\alpha_{\min }$ and $\alpha=\alpha_{\max }$. The shape of $f(\alpha)$ can be captured to some extent by the width 

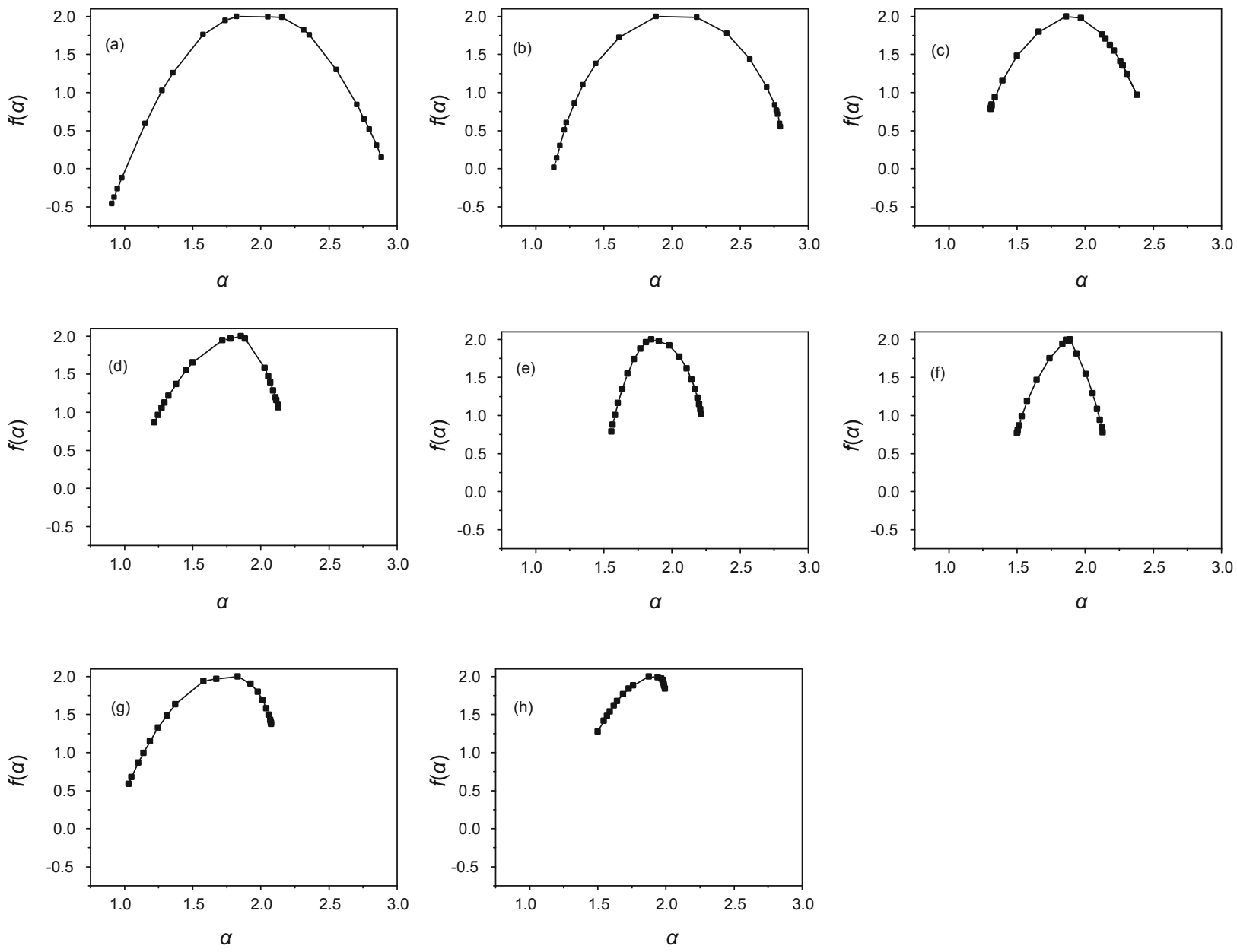

Fig. 6 Multifractal spectra versus $Q_{\mathrm{o}}$ at inclination $45^{\circ}$ and $Q_{\mathrm{w}}=0.83 \mathrm{~m}^{3} / \mathrm{h}$

(a) $Q_{\mathrm{o}}=0.45 \mathrm{~m}^{3} / \mathrm{h}$, PS; (b) $Q_{\mathrm{o}}=0.83 \mathrm{~m}^{3} / \mathrm{h}, \mathrm{PS}$; (c) $Q_{\mathrm{o}}=1.67 \mathrm{~m}^{3} / \mathrm{h}$, PS; (d) $Q_{\mathrm{o}}=2.50 \mathrm{~m}^{3} / \mathrm{h}, \mathrm{PS}$;

(e) $Q_{\mathrm{o}}=3.30 \mathrm{~m}^{3} / \mathrm{h}$, PS; (f) $Q_{\mathrm{o}}=4.00 \mathrm{~m}^{3} / \mathrm{h}, \mathrm{CT}$; (g) $Q_{\mathrm{o}}=5.83 \mathrm{~m}^{3} / \mathrm{h}, \mathrm{CT}$; (h) $Q_{\mathrm{o}}=7.50 \mathrm{~m}^{3} / \mathrm{h}$, TF

of the multifractal spectrum $\Delta \alpha=\alpha_{\max }-\alpha_{\min }$ and the difference of fractal dimensions $\Delta f=f\left(\alpha_{\max }\right)-f\left(\alpha_{\min }\right)$. We will discuss the dependence of $\Delta \alpha$ and $\Delta f$ with respect to the oil flow rate.

\subsection{The dependence of $\Delta \alpha$ with respect to $Q_{0}$}

In the multifractal analysis, $\alpha_{\min }$ is related to the maximum probability measure through $P_{\max } \sim \varepsilon^{a_{\min }}$, where $\varepsilon$ represents the measure approaching zero, whereas $\alpha_{\max }$ is related to the minimum probability measure by $P_{\min } \sim \varepsilon^{a_{\max }}$. The range of the probability measures can be described by the width of the spectrum $\Delta \alpha$ :

$$
P_{\max } / P_{\min } \sim \varepsilon^{-\Delta a}
$$

The greater the $\Delta \alpha$ value becomes, the wider the probability distribution is and the more complicated the countercurrent mode of the inclined oil-water two-phase flow is.

Fig. 8 illustrates the relationship between $\Delta \alpha$ and $Q_{0}$ at the same $Q_{\mathrm{w}}$. We find that the value of $\Delta \alpha$ gradually decreases with an increase in $Q_{\mathrm{o}}$ in the PS flow pattern, which indicates that the internal flow characteristics become more regular with the increase in $Q_{0}$. In the PS flow pattern, the oil phase exists in the form of intermittent oil swarms at the top of the pipe, while the water phase exists as a continuous phase at the bottom of the pipe and local countercurrent water flow also exists. In low $Q_{0}$, the oil swarms structure should be small, exhibiting quasi-periodic properties in the upper side, while the interval distance between two oil swarms is large so that the interval time should be more irregular. With an increase in $Q_{0}$, both the interval distance and time between oil swarms will become shorter and shorter, that is, the oil phase in the upper side of the pipe gradually tends to become continuous. The countercurrent water flow also exhibits a similar interval property at the bottom of the pipe with changing $Q_{0}$. So with an increase in $Q_{0}$, the width of the multifractal spectrum gradually decreases, and the probability distribution tends to be smaller, indicating the intermittent oil swarms tend to become continuous.

As can be seen in Fig. 8, we find, for the CT flow pattern the value of $\Delta \alpha$ also decreases with an increase in $Q_{0}$. On the basis of the PS flow pattern, with a further increase in $Q_{0}$, the interval characteristics between oil swarms will disappear and the oil phase will form a continuous flow at the top of the pipe, at this time the flow pattern will evolve into a CT flow pattern. With an increase in $Q_{0}$, the interval between water- 

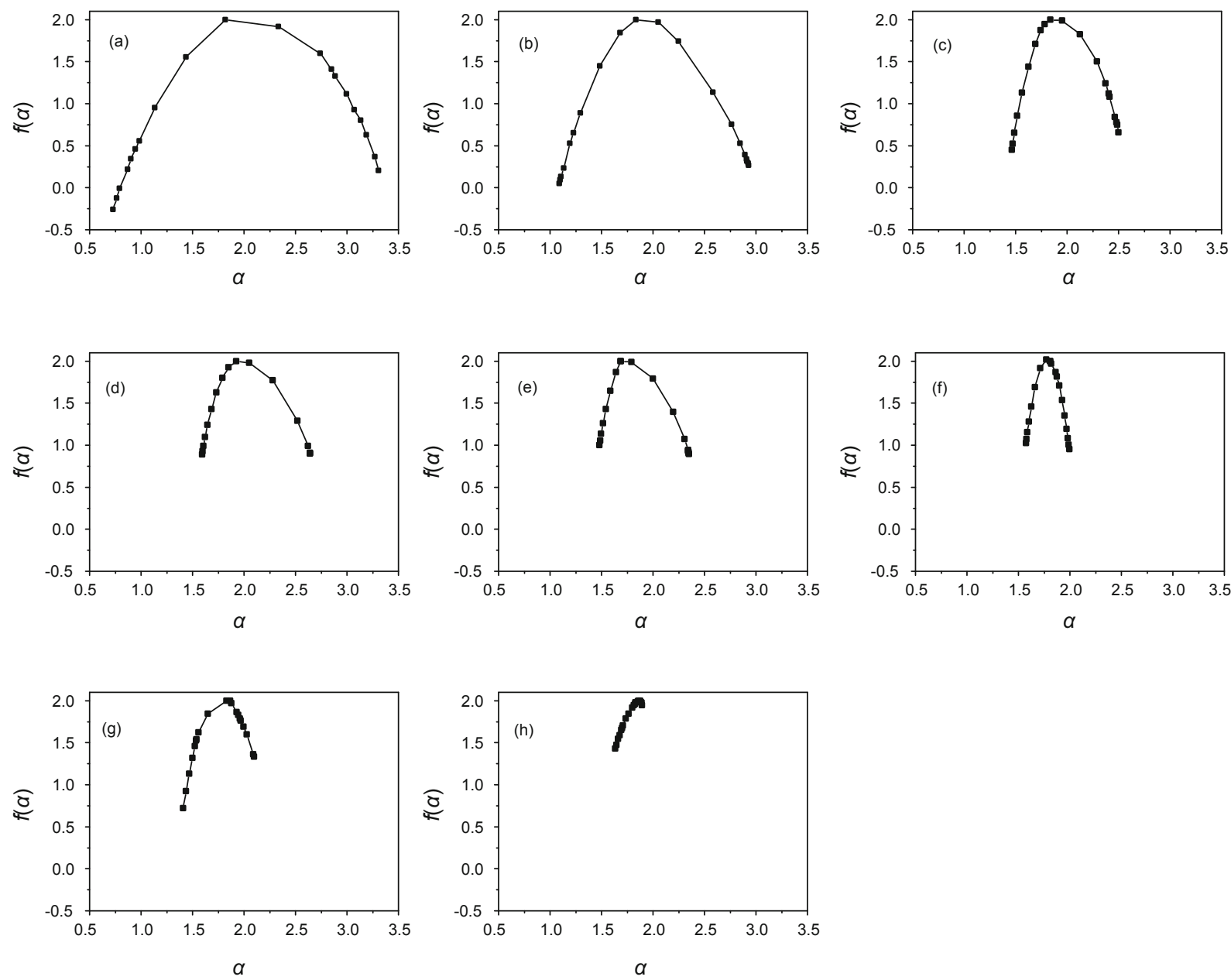

Fig. 7 Multifractal spectra versus $Q_{\mathrm{o}}$ at inclination $45^{\circ}$ and $Q_{\mathrm{w}}=1.64 \mathrm{~m}^{3} / \mathrm{h}$

(a) $Q_{0}=0.45 \mathrm{~m}^{3} / \mathrm{h}, \mathrm{PS}$; (b) $Q_{\mathrm{o}}=0.83 \mathrm{~m}^{3} / \mathrm{h}$, PS; (c) $Q_{\mathrm{o}}=1.67 \mathrm{~m}^{3} / \mathrm{h}, \mathrm{PS}$; (d) $Q_{\mathrm{o}}=2.50 \mathrm{~m}^{3} / \mathrm{h}, \mathrm{PS}$;

(e) $Q_{\mathrm{o}}=3.30 \mathrm{~m}^{3} / \mathrm{h}, \mathrm{PS}$; (f) $Q_{\mathrm{o}}=4.00 \mathrm{~m}^{3} / \mathrm{h}, \mathrm{CT}$; (g) $Q_{\mathrm{o}}=5.83 \mathrm{~m}^{3} / \mathrm{h}, \mathrm{CT}$; (h) $Q_{\mathrm{o}}=8.30 \mathrm{~m}^{3} / \mathrm{h}, \mathrm{TF}$

countercurrent flows will decrease and the countercurrent flow of the oil droplets will also tend to become continuous, so all of these will lead to a decrease in the width of the multifractal spectrum. However, compared with the PS flow pattern, the motion mode of the CT flow pattern is simpler; consequently, the value of $\Delta \alpha$ for CT flow pattern is smaller than that for PS flow pattern.

Otherwise, the value of $\Delta \alpha$ for the TF flow pattern is the smallest among three inclined oil-water flow patterns. As we have mentioned above, the alternation of oil-dominated and water-dominated flow structure is the main characteristics. So compared with other flow patterns, the TF flow pattern performs a relatively single motion mode, so the width of multifractal spectrum is very narrow, which to some extent means that this flow pattern is close to monofractal instead of multifractal characteristics.

The observed multifractal behavior can be partly interpreted in terms of the spatial intermittency (Argoul et al, 1989; Kuramoto and Nakao, 1997) and the origin of the multifractal hidden in the inclined oil-water two-phase flow may be the long range correlations of the intermittent fluctuation (Mandelbrot, 1990; Mordant et al, 2002). The self-similarity property in these flow patterns results from the countercurrent structure and intermittency of oil swarms, which is the combination of mass transfer and fluid dynamics. Otherwise, in oil swarm growth and coalescence, the oil phase transferred from small swarms (with low $Q_{\mathrm{o}}$ ) into big ones (with high $Q_{\mathrm{o}}$ ), forming a continuous structure, which may be the origin of long-range correlation.

\subsection{The dependence of $\Delta f$ with respect to $Q_{0}$}

The difference of fractal dimensions $\Delta f$ is also a very important parameter in characterizing multifractal properties. The $f\left(\alpha_{\min }\right)$ value reflects the most concentrated distribution of probability measure with $N_{P_{\max }}=N_{\alpha_{\min }} \sim \varepsilon^{-f\left(\alpha_{\min }\right)}$, while $f\left(\alpha_{\max }\right)$ value reflects the rarest distribution of probability measure with $N_{P_{\min }}=N_{\alpha_{\max }} \sim \varepsilon^{-f\left(\alpha_{\max }\right)}$. Hence, the value of $\Delta f$ can describe the ratio between the fractal dimensions of the maximum and the minimum growth probability,

$$
N_{P_{\max }} / N_{P_{\min }} \sim \varepsilon^{\Delta f}
$$

Fig. 9 depicts the dependence of $\Delta f$ with respect to $Q_{\text {o }}$ at the same $Q_{\mathrm{w}}$. We find that the difference of the fractal dimensions gradually decreases with an increase in $Q_{\mathrm{o}}$ in 

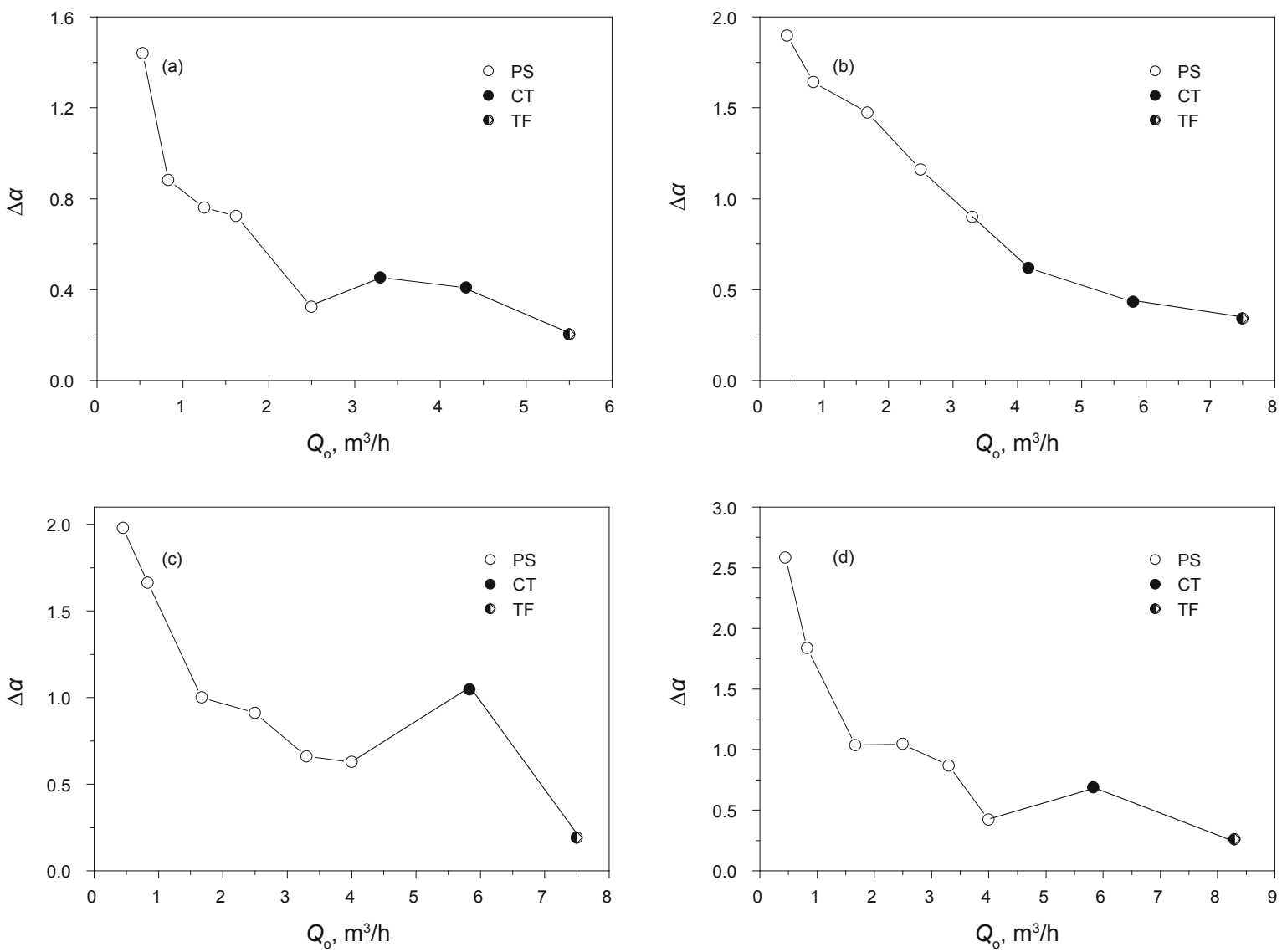

Fig. 8 The width of spectrum $\Delta \alpha$ versus $Q_{\text {。 }}$

(a) $Q_{\mathrm{w}}=0.21 \mathrm{~m}^{3} / \mathrm{h}$; (b) $Q_{\mathrm{w}}=0.42 \mathrm{~m}^{3} / \mathrm{h}$; (c) $Q_{\mathrm{w}}=0.83 \mathrm{~m}^{3} / \mathrm{h}$; (d) $Q_{\mathrm{w}}=1.64 \mathrm{~m}^{3} / \mathrm{h}$

the PS flow pattern, which indicates that the concentrated regions of the probability distribution become more common and the internal flow characteristics become more regular. With an increase in $Q_{0}$, both interval distance and time between oil swarms will become shorter and shorter, that is, the oil phase in the upper side of the pipe gradually tends to become continuous. On the other hand, with increasing $Q_{\mathrm{o}}$ the resistance to the motion of oil swarms gradually increases due to the increase in the equivalent viscosity of the oil-water mixture, because the oil viscosity is much higher than the water viscosity. So the motion of the fluid in the whole pipe tends to be regular; consequently, the $\Delta f$ value of the PS flow pattern decreases with increasing $Q_{0}$.

Especially in Fig. 9(d), the $\Delta f$ values are negative when $Q_{\text {o }}$ ranges from 3.30 to $4.30 \mathrm{~m}^{3} / \mathrm{h}$, indicating that there are more concentrated regions than low-density sites. This phenomenon only takes place at the boundary of PS and CT flaw patterns at higher $Q_{\mathrm{w}}$, and the $|\Delta f|$ values are still low, indicating that at high $Q_{\mathrm{o}}$ and $Q_{\mathrm{w}}$ the local countercurrent structure in the pipe is almost unchanged and the flow mechanism is almost the same.

Likewise for the CT flow pattern, the $\Delta f$ value also decreases with increasing $Q_{0}$, indicating that the concentrated regions of the probability distribution become more numerous. We infer that with increasing $Q_{0}$, the equivalent viscosity of the oil-water mixture increases, correspondingly, the resistance to the motion increases in the pipe; consequently, the internal flow characteristics become more regular for the $\mathrm{CT}$ flow pattern, which can be reflected by the decrease in $\Delta f$.

For the TF flow pattern with a further increase in $Q_{\mathrm{o}}$ in the pipe, the equivalent viscosity of the oil-water mixture continues increasing compared with other flow patterns, and both oil and water phases are continuous instead of intermittent; consequently, the probability distribution is concentrated and the $\Delta f$ value is large. However, due to the narrow form of the singularity spectrum, the $\Delta f$ values of the TF flow pattern are not the largest.

\subsection{The dependence of $\Delta \alpha / \Delta f$ with respect to flow pattern identification}

The $\Delta \alpha$ and $\Delta f$ values in the multifractal analysis can effectively reflect the evolutionary process of the flow patterns. Note that a single characteristic quantity can not be used to analyze all flow conditions; therefore we define a variable ratio as $\Delta \alpha / \Delta f$ to describe the different multifractal spectrum mode and further to characterize the two-phase flow.

The distributions of $\Delta \alpha / \Delta f$ for different flow conditions are shown in Fig. 10. We can see that the $\Delta \alpha / \Delta f$ values are 2.11105.2 for PS flow, 0.75-1.12 for CT flow, and 0.26-0.51 for TF flow, respectively. The distribution intervals of the three flow patterns reflect their particular modes: the multifractal spectrum of the PS flow exhibits a near symmetric spectrum shape, correspondingly, its $\Delta \alpha / \Delta f$ value is relatively large i.e., greater than 2; the multifractal spectrum of the CT flow 

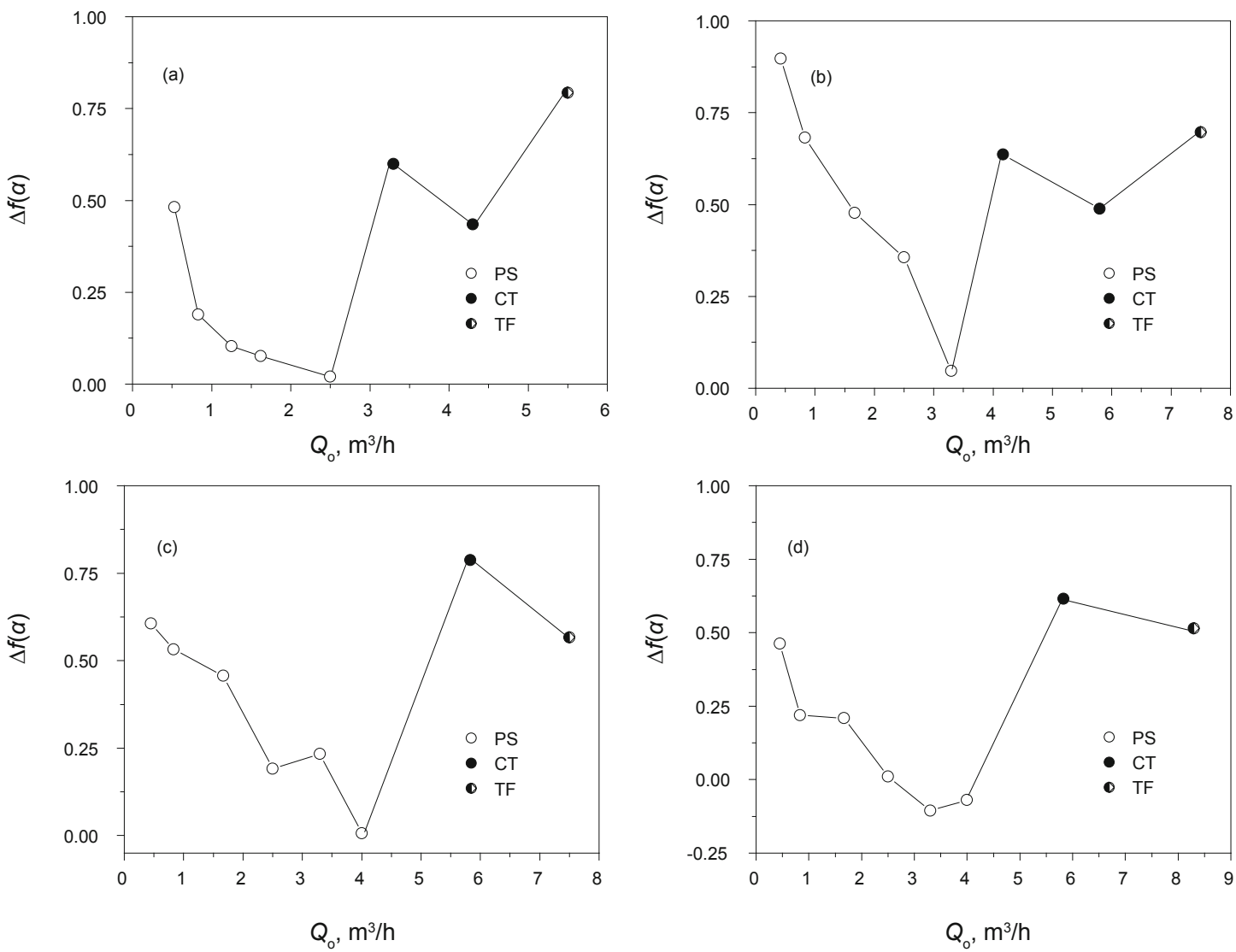

Fig. 9 The difference of fractal dimension $\Delta f$ versus $Q_{\text {o }}$

(a) $Q_{\mathrm{w}}=0.21 \mathrm{~m}^{3} / \mathrm{h}$; (b) $Q_{\mathrm{w}}=0.42 \mathrm{~m}^{3} / \mathrm{h}$; (c) $Q_{\mathrm{w}}=0.83 \mathrm{~m}^{3} / \mathrm{h}$; (d) $Q_{\mathrm{w}}=1.64 \mathrm{~m}^{3} / \mathrm{h}$

exhibits a left-hooked shape, correspondingly, the $\Delta \alpha / \Delta f$ value at the second place; but the $\Delta \alpha / \Delta f$ value of the TF flow is the lowest, less than 0.5 , and a single edge shape is observed for this multifractal spectrum. Therefore, we can effectively identify three inclined oil-water flow patterns by using the values of $\Delta \alpha / \Delta f$.

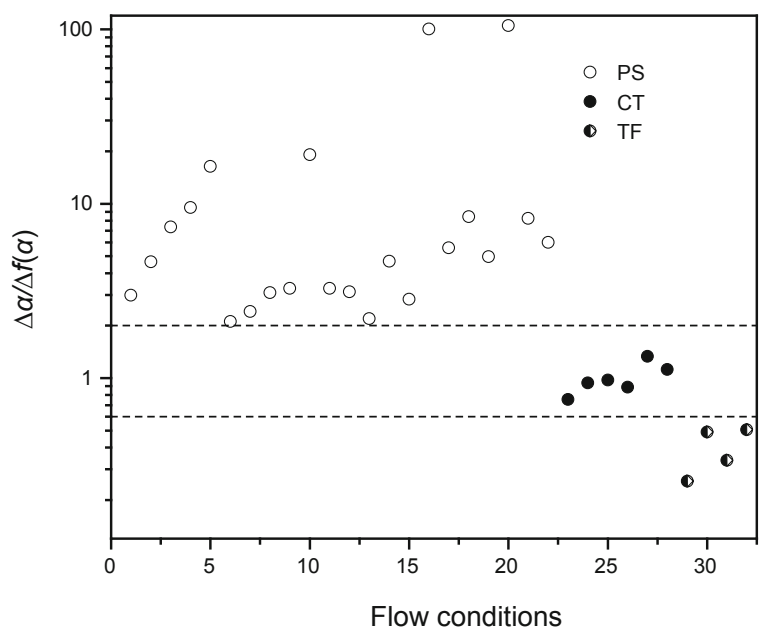

Fig. 10 The distribution of $\Delta \alpha / \Delta f$ for different flow conditions

\section{Conclusions}

Inclined oil-water two-phase flow is a complex dynamic system. In this paper we use long-range correlation and multifractal spectra to probe the nonlinear mechanisms underlying the flow transitions.

Magnitude and sign decomposition analysis can uncover the long-range correlation properties of the original time series. The magnitude increment series of different flow patterns reflect different positive correlation properties. In particular, for different flow patterns, the sign increment series at short time scales shows the strong and similar positive correlation, but at long time scales it presents anticorrelation properties with certain differences.

Inclined oil-water two-phase flows present typical multifractal properties, and the singularity spectra show different scaling properties at different scales, which can reveal dynamic complexity and fluid mechanisms for different flow patterns. Our results can provide clues for understanding the fluid dynamics in the transitions of flow patterns from different time scales.

\section{Acknowledgements}

This work was supported by the National Natural Science Foundation of China (Grant Nos. 41174109, 61104148, 50974095) and the National Science and Technology Major Projects (Grant No. 2011ZX05020-006).

\section{References}

Amitrano C, Coniglio A and di Liberto F. Growth probability distribution in kinetic aggregation processes. Physical Review Letters. 1986. 
57(3): 1016-1019

Argoul F, Arneodo A, Grasseau G, et al. Wavelet analysis of turbulence reveals the multifractal nature of the Richardson cascade. Nature. 1989. 338: 51-53

Ashkenazy Y, Ivanov P C, Havlin S, et al. Magnitude and sign correlations in heartbeat fluctuations. Physical Review Letters. 2001. 86(9): 1900-1903

Chhabra A B and Sreenivasan K R. Negative dimensions: theory, computation and experiment. Physical Review A. 1991. 43: 11141117

Daw C S, Finney C E A, Vasudevan M, et al. Self-organization and chaos in a fluidized bed. Physical Review Letters. 1995. 75(12): 2308-2311

Flores J G, Chen X T, Sarica C, et al. Characterization of oil-water flow patterns in vertical and deviated wells. SPE Production \& Facilities. 1999. 14(2): 94-101

Gao Z K, Jin N D, Wang W X, et al. Motif distributions of phase-space networks for chaotic systems and application to experimental twophase flow patterns. Physical Review E. 2010. 82(1): 016210-1-8

Gong J, Wang W and Yu D. Experimental study on flow patterns of heavy oil-water two-phase flow in horizontal pipes. Acta Petrolei Sinica. 2007. 28(2): 140-143 (in Chinese)

Hill A D and Oolman T. Production logging tool behavior in two-phase inclined flow. Journal of Petroleum Technology. 1982. 34: 2432-2440

Jin N D, Xin Z, Wang, J, et al. Design and geometry optimization of conductivity probe with a vertical multiple electrode array for measuring volume fraction and axial velocity of two-phase flow. Measure Science and Technology. 2008. 19(4): 045403-1-19

Kantelhardt J W, Zschiegner S A, Koscielny-Bunde E, et al. Multifractal detrended fluctuation analysis of nonstationary time series. Physica A. 2002. 316: 87-114

Kumara W A S, Halvorsen B M and Melaaen M C. Particle image velocimetry for characterizing the flow structure of oil-water flow in horizontal and slightly inclined pipes. Chemical Engineering Science. 2010. 65: 4332-4349

Kuramoto Y and Nakao H. Scaling properties in large assemblies of simple dynamical units driven by long wave random forcing. Physical Review Letters. 1997. 78: 4039-4042

Mandelbrot B B. Negative fractal dimensions and multifractals. Physica A. 1990. 163: 306-315

Mordant N, Delour J, Leveque E, et al. Long time correlations in Lagrangian dynamics: a key to intermittency in turbulence. Physical Review Letters. 2002. 89(25): 254502-1-4

Peng C K, Havlin S, Stanley H E, et al. Quantification of scaling exponents and crossover phenomena in non stationary heartbeat time series. Chaos: An Interdisciplinary Journal of Nonlinear Science. 1995. 5(1): 82-87

Rodriguez O M H and Baldani L S. Prediction of pressure gradient and holdup in wavy stratified liquid-liquid inclined pipe flow. Journal of Petroleum Science and Engineering. 2012. 96: 140-151

Strazza D, Grassi B, Demori M, et al. Core-annular flow in horizontal and slightly inclined pipes: existence, pressure drops, and hold-up. Chemical Engineering Science. 2011. 66: 2853-2863

Wang H T, Zhang L H, Guo J J, et al. An efficient algorithm to compute transient pressure responses of slanted wells with arbitrary inclination in reservoirs. Petroleum Science. 2012. 9(2): 212-222

Yang M, Wu X L, Wang Z L, et al. Measurement of oil-water flow patterns by invading production logging tools in inclined wells. Science Technology Review. 2008. 26(15): 73-78 (in Chinese)

Zhu L, Jin N D, Gao Z K et al. Multi-scale cross entropy analysis for inclined oil-water two-phase countercurrent flow patterns. Chemical Engineering Science. 2011. 66: 6099-6108

(Edited by Sun Yanhua) 